\title{
Healing the injured vessel wall using microRNA-facilitated gene delivery
}

\author{
Mark W. Feinberg \\ Department of Medicine, Cardiovascular Division, Brigham and Women's Hospital, Harvard Medical School, Boston, Massachusetts, USA.
}

Drug-eluting stents have emerged as potent weapons in the treatment of patients with symptomatic coronary artery disease by reducing restenosis rates; however, a significant clinical consequence of these stents is delayed reendothelialization, which may increase the risk of late stent thrombosis. In this issue of the $\mathrm{JCl}$, Santulli and colleagues generated an adenovirus that expresses the cyclin-dependent kinase inhibitor p27 ${ }^{\text {Kip } 1}$ (p27) and bears four tandem copies of target sequences for the endothelial cell-enriched microRNA (miRNA) miR-126-3p (Ad-p27-126TS) in an attempt to specifically reduce proliferation of vascular smooth muscle cells, but not endothelial cells. Indeed, delivery of Ad-p27-126TS to balloon-injured arteries in rats not only induced faster and more complete reendothelialization, but also effectively improved neointimal hyperplasia, hypercoagulability, and vasoreactivity. Collectively, these findings provide a cogent foundation for the potential therapeutic use of miRNA-facilitated gene delivery strategies to heal vessel wall injury.

\section{Vascular endothelium and injury from percutaneous coronary intervention}

Over the past 37 years, percutaneous coronary intervention (PCI) has revolutionized patient revascularization care for symptomatic ischemic coronary artery disease by improving myocardial perfusion and clinical outcomes. However, PCI has been considered a double-edged sword due to the endothelial injury and denudation that occurs from a combination of high-pressure balloon inflations and relatively noncompliant stent struts. The vascular response to mechanical endothelial cell (EC) and smooth muscle cell (SMC) injury represents a complex series of molecular and cellular events that involve the orchestration between resident and recruited vascular cells, leukocytes, and progenitor cells that regulate the ensuing neointimal hyperplasia, vascular remodeling, and reendothelialization (1). A range of innovative therapies and mechanical coronary device strategies have evolved to improve the outcomes and response to injury induced as a consequence of these procedures for patients with symptomatic coronary lesions. By 1993, baremetal stents (BMS) were FDA approved in the US. Compared with balloon angioplasty alone, BMS dramatically improved vessel remodeling with reduced restenosis rates and fewer subsequent reinterventions required. The improvements seen with BMS are thought to be primarily due to a reduction in late lumen loss, a process known as negative remodeling (2); however, clinical restenosis rates still occurred in approximately $20 \%$ to $30 \%$ of patients receiving BMS, in part, due to persistent neointimal hyperplasia $(3,4)$. A decade later, drug-eluting stents (DES), which release antimitotic drugs, such as sirolimus, paclitaxel, zotarolimus, or everolimus, were developed and potently inhibit SMC proliferation and extracellular matrix generation, resulting in

Related Article: p. 4102

Conflict of interest: The author has declared that no conflict of interest exists.

Reference information: / Clin Invest. 2014;124(9):3694-3697. doi:10.1172/JCI77509.

markedly reduced neointimal hyperplasia and an approximately $70 \%$ reduction of coronary restenosis compared with $\mathrm{BMS}(5,6)$.

Because DES-dependent drug delivery does not discriminate between proliferating vascular SMCs (VSMCs) and ECs, delayed reendothelialization has been considered a major limiting factor for optimal vascular repair and may predispose patients to late thrombotic events ( 7 , 8). Indeed, preclinical studies and pathologic evaluation at the time of human autopsy have confirmed a delayed reendothelialization associated with $\operatorname{DES}(9,10)$. Considerable attention has been focused on the possibility of late (30 days to 1 year) and very late (after 1 year) thrombosis associated with DES and whether extended dual antiplatelet therapy (DAPT) reduces this potential thrombotic risk (11-13). DAPT itself may also carry higher bleeding risks over this extended period. Every year, nearly 500,000 patients in the US undergo PCI for symptomatic coronary artery disease, and DES are deployed in at least $75 \%$ of these cases (14). As such, identification of novel strategies to restore rapid reendothelialization may allow for improved clinical outcomes and simultaneously minimize the need for prolonged DAPT.

\section{Preclinical and clinical reendothelialization strategies}

An intact vascular endothelium serves not only as a functional barrier between blood and all tissues, but also dictates the vasoreactive, thrombogenic, inflammatory, and adhesive properties that are important for the vessel response to injury. Reendothelialization after PCI is thought to occur, in part, from residual islands of noninjured ECs within the stented region, flanking ECs outside of the stented region, and circulating or resident progenitor cells (7). While BMS and DES have addressed arterial remodeling and neointimal hyperplasia, the incomplete endothelialization associated with these stents remains an 

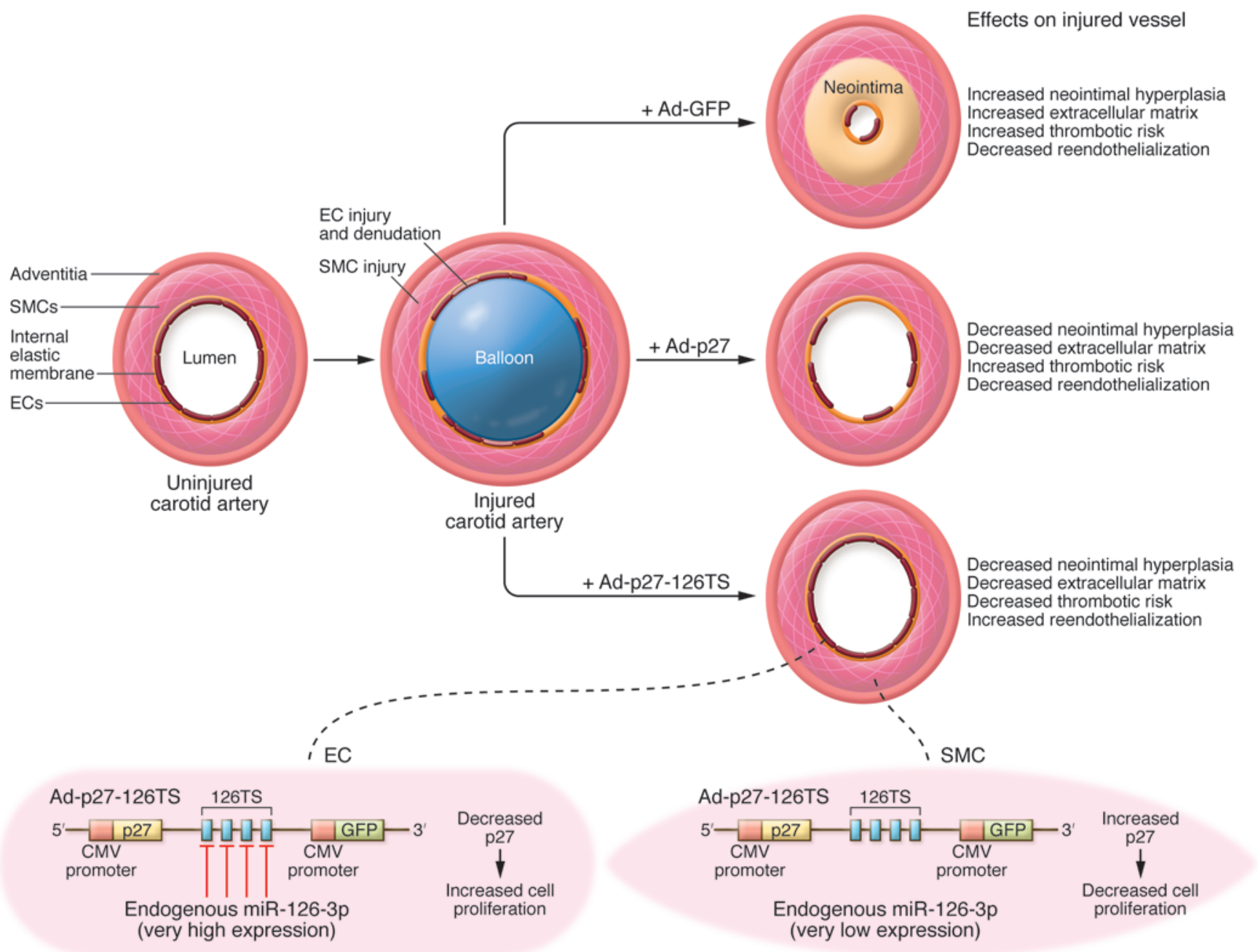

Figure 1. miRNA-facilitated gene delivery of p27 to the injured vessel wall reduces neointimal hyperplasia and improves reendothelialization. Carotid artery injury, such as occurs following balloon inflation, results in neointimal hyperplasia, increased extracellular matrix deposition, and loss of vessel endothelium, all of which increase thrombotic risk. Treatment with adenovirus control (Ad-GFP) does not alter injury-induced vessel damage. Following carotid artery injury, adenoviral delivery of an adenovirus for p27 (Ad-27) inhibits neointimal hyperplasia, but does not improve reendothelialization. Santulli and colleagues (21) have demonstrated that local delivery of an adenovirus bearing four tandem copies of target sequences for miR-126-3p (Ad-p27-126TS) reduces VSMC proliferation, but not EC proliferation. Because of the markedly higher expression levels of endogenous miR-126-3p in ECs compared with levels in VSMCs, adenoviral p27 overexpression is suppressed in ECs via miR-126-3p-mediated binding of complementary target sequences in the Ad-p27-126TS vector. Consequently, neointimal hyperplasia is reduced and endothelial growth and coverage are increased, leading to improved thrombotic risk and vasoreactivity.

active area of investigation with renewed scientific interest, given the potential for late thrombotic risk. Additional factors, such as delayed hypersensitivity reactions to the DES polymer or technical and mechanical factors (6), also likely contribute to stent thrombosis independently of reendothelialization. Nevertheless, a range of preclinical and clinical strategies have emerged in an attempt to address both reendothelialization and antithrombotic risk. These strategies include recruitment of circulating progenitor cells, delivery of proangiogenic factors, and alteration of DES properties, such as drug dose/release kinetics, stent alloy, architecture, polymer coating composition, or abluminal release of antimitotic drugs, among others $(6,15$, 16). For example, the Genous (OrbusNeich) stent, designed to capture so-called CD34 endothelial progenitor cells, which include both endothelial and nonendothelial progenitor subsets, demonstrated promising reendothelialization properties and reduced neointimal hyperplasia in preclinical studies (17). Unfortunately, compared with the Taxus (Boston Scientific) DES, the Genous stent showed no major differences in clinical events or target vessel revascularization out to two years (18).
Viral-based gene strategies have been used to transduce the vessel wall with a range of cell-cycle regulators and have provided scientific insights and therapeutic opportunities for regulating neointimal hyperplasia (19, 20); however, lack of cell specificity also adversely affects the growth of the neoendothelium. In this issue of JCI, Santulli, et al. (21) created a unique adenovirus that expresses the cyclin-dependent kinase inhibitor p27 $7^{\text {Kip1 }}$ (p27) under a strong CMV promoter and bears four tandem copies of target sequences for the EC-enriched microRNA (miRNA) miR-126-3p (Ad-p27-126TS) in 
an attempt to specifically reduce proliferation of VSMCs, but not ECs (Figure 1). miRNAs are evolutionarily conserved, single-stranded, noncoding RNAs that suppress protein expression by binding to target complementary sequences in the $3^{\prime}$-UTRs of mRNAs, an event that is directed by the "seed region" nucleotides 2-7 at the 5 ' end of the mature miRNA (22). Therapeutic manipulation of miRNAs has recently garnered scientific interest for their contribution to a number of vascular disease states $(23,24)$. The concept of miRNA-facilitated viral delivery used by Santulli et al. (21) takes advantage of the well-established observation that miR126-3p expression is robustly enriched in the vascular endothelium and nearly absent in VSMCs under normal homeostatic conditions (25). As such, endogenous miR-126-3p binding to the Ad-p27$126 \mathrm{TS}$ would diminish the ability of p27 to be overexpressed in ECs, while being permissive to p27 overexpression in SMCs, thereby inducing growth arrest in VSMCs, but not in ECs, and blocking VSMC-mediated neointimal proliferation. Indeed, using both in vitro and in vivo studies, Santulli and colleagues found that Ad-p27126TS transduction in VSMCs blocks cell proliferation and migration, while protecting EC functional properties (21). In a balloon-injured rat carotid injury model, local delivery of Ad-p27-126TS (injected into the common carotid artery and allowed to incubate for 20 minutes in the absence of flow) potently reduced neointimal hyperplasia to the same extent as that of Ad-p27 without the miR-126-3p target sequences. Importantly, after two weeks, injured vessels transduced with Ad-p27126TS exhibited nearly $80 \%$ reendothelial coverage ( $88 \%$ by 4 weeks) compared with that observed in uninjured vessels, whereas the Ad-p27-transduced vessels showed only $26 \%$ EC coverage ( $29 \%$ by 4 weeks) (Figure 1). These findings were verified using a combination of immunohistochemistry for VE-cadherin on en face and cross-sectional preparations, tri- and bidimensional confocal microscopy, and scanning electron microscopy. Consistent with an intact functional EC barrier, Ad-p27-126TS reduced accumulation of CD $45^{+}$leukocytes and arterial structure for collagen and elastin fibers. Finally, Ad-p27-126TS delivery also significantly improved hypercoagulability, reflected by circulating levels of plasma D-dimer and phenylephrine-induced vascular reactivity, to the same extent exhibited by uninjured vessels after two weeks.

\section{Conclusions and future directions}

Taken together, the work by Santulli et al. (21) provides compelling evidence for further exploration into the incorporation of miRNA target sites to facilitate gene delivery in a cell-specific manner for improving reendothelialization after vessel wall injury. Because endothelial repair may be a limiting factor in a range of vascular disease states, these findings may provide therapeutic opportunities for related conditions, such as vascular graft failure, which may develop in patients requiring arteriovenous fistulae for hemodialysis, vascular conduits for peripheral artery disease, or saphenous vein grafts for coronary artery bypass graft surgery, among others. Nevertheless, several important questions and issues remain before this innovative technology is ready to translate to human studies. First, vascular carotid injury in rats does not necessarily recapitulate human coronary injury due to anatomical considerations. For example, rats exhibit lower medial wall elastin, a smaller subintimal layer, and a more continuous internal elastic lamina (26); therefore, better insights into vessel injury could be provided by larger animals such as pigs, which more closely resemble the human response to coronary artery injury (27). Second, it is unclear whether Ad-p27-126TS will function as effectively under harsher environmental conditions such as hypercholesterolemia, diabetes, or native atherosclerosis, conditions that may alter endogenous miR-126-3p expression in the vascular endothelium, thereby allowing for some p27 overexpression in ECs and potentially abrogating reendothelialization. Third, in this study, local adenoviral-mediated arterial delivery required cessation of blood flow for 20 minutes; therefore, alternative therapeutically compatible types of delivery approaches will need to be considered for PCI, though this type of local delivery may be feasible for more accessible surgical vascular grafts, delivered either endoluminally or periadventitially.
Fourth, the effect of Ad-p27-126TS on other resident cell types, such as adventitial fibroblasts and leukocyte subsets, should be further explored. Fifth, investigation of Ad-p27-126TS efficacy in the presence of BMS would be informative, as reendothelialization will also be affected by other stent-associated features such as local hemodynamic shear forces. Sixth, recent findings suggest that miR-126 may be secreted from ECs and transmitted to SMCs under specific shear stress conditions in vitro and therefore may attenuate cell-specific effects in vivo (28). Seventh, while mechanistic studies have demonstrated that sirolimus treatment can induce p27 in cells (29), viral-mediated overexpression of p27 (or other cell-cycle regulators) may not recapitulate the same growth inhibitory properties. Finally, there are concerns about the chronic effects of Ad-p27-126TS due to its potential to function as a "sponge" and deplete endogenous miR-126-3p in ECs. Santulli et al. (21) addressed the potential of Ad-p27-126TS to regulate endogenous miR-126-3p and found only a modest reduction of endogenous miR126-3p expression in ECs and no significant effect on the miR-126-3p target gene PIK3R2 or the related miR-126-5p, which has been shown to facilitate EC recovery after injury (30). These findings are consistent with the specificity of other miRNA "sponges" that have been used to regulate gene and miRNA expression in other cellular systems $(31,32)$. Future studies to address some of these issues regarding Ad-p27-126TS will provide important insights and define whether miRNA-facilitated gene delivery will promote reendothelialization, particularly in larger animals, and be transformative for healing the injured vessel wall with PCI or for other related vascular disease states.

\section{Acknowledgments}

This work was supported by funding from the NIH (HL115141 and HL117994).

Address correspondence to: Mark W. Feinberg, Department of Medicine, Cardiovascular Division, Brigham and Women's Hospital, Harvard Medical School, 77 Avenue Louis Pasteur, NRB-742F, Boston, Massachusetts 02115, USA. Phone: 617.525.4381; E-mail: mfeinberg@partners.org. 
1. Inoue T, Croce K, Morooka T, Sakuma M, Node $\mathrm{K}$, Simon DI. Vascular inflammation and repair: implications for re-endothelialization, restenosis, and stent thrombosis. JACC Cardiovasc Interv. 2011;4(10):1057-1066.

2. Lafont A, Guzman LA, Whitlow PL, Goormastic M, Cornhill JF, Chisolm GM. Restenosis after experimental angioplasty. Intimal, medial, and adventitial changes associated with constrictive remodeling. Circ Res. 1995;76(6):996-1002.

3. Fischman DL, et al. A randomized comparison of coronary-stent placement and balloon angioplasty in the treatment of coronary artery disease. Stent Restenosis Study Investigators. NEngl J Med. 1994;331(8):496-501.

4. Macaya C, et al. Continued benefit of coronary stenting versus balloon angioplasty: one-year clinical follow-up of Benestent trial. Benestent Study Group. J Am Coll Cardiol. 1996;27(2):255-261.

5. Stettler C, et al. Outcomes associated with drug-eluting and bare-metal stents: a collaborative network meta-analysis. Lancet. 2007;370(9591):937-948.

6. Stefanini GG, Holmes DR, Holmes DR Jr. Drug-eluting coronary-artery stents. $\mathrm{NEnglJ}$ Med. 2013;368(3):254-265.

7. Luscher TF, et al. Drug-eluting stent and coronary thrombosis: biological mechanisms and clinical implications. Circulation. 2007;115(8):1051-1058.

8. Garg P, Mauri L. The conundrum of late and very late stent thrombosis following drug-eluting stent implantation. Curr Opin Cardiol. 2007;22(6):565-571.

9. Kotani J, et al. Incomplete neointimal coverage of sirolimus-eluting stents: angioscopic findings. J Am Coll Cardiol. 2006;47(10):2108-2111.

10. Joner M, et al. Pathology of drug-eluting stents in humans: delayed healing and late thrombotic risk. J Am Coll Cardiol. 2006;48(1):193-202.

11. Lagerqvist B, et al. Stent thrombosis in Sweden: a report from the Swedish Coronary Angiography and Angioplasty Registry. Circ Cardiovasc Interv. 2009;2(5):401-408.
12. McFadden EP, et al. Late thrombosis in drug-eluting coronary stents after discontinuation of antiplatelet therapy. Lancet. 2004;364(9444):1519-1521.

13. Mauri L, et al. Rationale and design of the dual antiplatelet therapy study, a prospective, multicenter, randomized, double-blind trial to assess the effectiveness and safety of 12 versus 30 months of dual antiplatelet therapy in subjects undergoing percutaneous coronary intervention with either drug-eluting stent or bare metal stent placement for the treatment of coronary artery lesions. Am Heart J. 2010;160(6):1035-1041.

14. Go AS, et al. Heart disease and stroke statistics 2014 update: a report from the American Heart Association. Circulation. 2014;129(3):e28-e292.

15. Palmerini T, et al. Clinical outcomes with bioabsorbable polymer- versus durable polymer-based drug-eluting and bare-metal stents: evidence from a comprehensive network meta-analysis. J Am Coll Cardiol. 2014;63(4):299-307.

16. Wara AK, et al. Bone marrow-derived Kruppellike factor 10 controls reendothelialization in response to arterial injury. Arterioscler Thromb Vasc Biol. 2013;33(7):1552-1560.

17. van Beusekom HM, Ertas G, Sorop O, Serruys PW, van der Giessen WJ. The Genous endothelial progenitor cell capture stent accelerates stent re-endothelialization but does not affect intimal hyperplasia in porcine coronary arteries. Catheter Cardiovasc Interv. 2012;79(2):231-242.

18. Beijk MA, et al. Genous endothelial progenitor cell capturing stent vs. the Taxus Liberte stent in patients with de novo coronary lesions with a high-risk of coronary restenosis: a randomized, single-centre, pilot study. Eur Heart J. 2010;31(9):1055-1064.

19. Nabel EG, Plautz G, Boyce FM, Stanley JC, Nabe GJ. Recombinant gene expression in vivo within endothelial cells of the arterial wall. Science. 1989;244(4910):1342-1344.

20. Tanner FC, et al. Differential effects of the cyclin-dependent kinase inhibitors p27(Kip1), p21(Cip1), and p16(Ink4) on vascular smooth muscle cell proliferation. Circulation. 2000;101(17):2022-2025.

21. Santulli G, et al. A selective microRNA-based strategy inhibits restenosis while preserving endothelial function. J Clin Invest. 2014;124(9):4102-4114.

22. Bartel DP. MicroRNAs: target recognition and regulatory functions. Cell. 2009;136(2):215-233.

23. Sun X, Belkin N, Feinberg MW. Endothelial microRNAs and atherosclerosis. Curr Atheroscler Rep. 2013;15(12):372.

24. Olson EN. MicroRNAs as Therapeutic Targets and Biomarkers of Cardiovascular Disease. Sci Transl Med. 2014;6(239):239ps233.

25. Wang S, et al. The endothelial-specific microRNA miR-126 governs vascular integrity and angiogenesis. Dev Cell. 2008;15(2):261-271.

26. Sims FH. A comparison of structural features of the walls of coronary arteries from 10 different species. Pathology. 1989;21(2):115-124.

27. Schwartz RS, Chronos NA, Virmani R. Preclinical restenosis models and drug-eluting stents: still important, still much to learn. JAm Coll Cardiol. 2004;44(7):1373-1385.

28. Zhou J, et al. Regulation of vascular smooth muscle cell turnover by endothelial cell-secreted microRNA-126: role of shear stress. Circ Res. 2013;113(1):40-51.

29. Moss SC, Lightell DJ, Lightell DJ Jr, Marx SO, Marks AR, Woods TC. Rapamycin regulates endothelial cell migration through regulation of the cyclin-dependent kinase inhibitor p27Kip1. J Biol Chem . 2010;285(16):11991-11997.

30. Schober A, et al. MicroRNA-126-5p promotes endothelial proliferation and limits atherosclerosis by suppressing Dlk1. Nat Med. 2014;20(4):368-376.

31. Kelly EJ, Hadac EM, Greiner S, Russell SJ. Engineering microRNA responsiveness to decrease virus pathogenicity. Nat Med. 2008;14(11):1278-1283.

32. Brown BD, Naldini L. Exploiting and antagonizing microRNA regulation for therapeutic and experimental applications. Nat Rev Genet. 2009;10(8):578-585. 